\title{
A License Plate Recognition Method for Community Monitor Based on Hausdorff Distance
}

\author{
Lingxian Yang * Heping Chen Wei Zhang \\ College of Information Science and Engineering,Wuhan University of Science and Technology, \\ Wuhan, P.R. China, 430081 \\ * Corresponding author's Email:yanglingxian@wust.edu.cn
}

\begin{abstract}
In this paper, a novel license plate recognition approach for community monitor using hausdorff distance is proposed. This method reduces the problem of rotation and translation transformation between the license plate and model rely on finding the minimizing the hausdorff distance between two point sets. The algorithm utilizes color and edge characters, meanwhile, combines some conventional image preprocessing methods to get several regions of interest, and the least trimmed square hausdorff dis-tance is computed to select the validity license plate and recognize the detail characters according to a well-organized digital models and the match threshold is defined in ad-vance. The result of algorithm shows some tolerant of small location errors occurring in region of interest that has good performance and efficiency.
\end{abstract}

Keywords: License plate recognition; Hausdorff distance; Community monitor; Character segmentation; Model match

\section{Introduction}

It is an important safeguard measure for community security to implement intelligent video monitoring [1], as well as automated license recognition is the key content to intelligent video monitoring geared to community. License plate is the unique identification for each car, so vehicle monitor and identification mean actually utilizing image processing, pattern recognition and etc. to realize plate segmentation and character recognition. And it also has a wide range of applications, such as criminal pursuit, community management, vehicle status checking, automatic toll collection, traffic flow magnitude control and etc. A complete LPR (License Plate Recognition) system generally consists of the following major steps [2], [3]: image collecting and pre-processing, license plate detection (to roughly find the regions of interest (ROI) from the input images); license plate segmentation (to separate symbols/characters from each other); character recognition (to identify symbols/characters from image by predefined recognition models). In this paper, we propose a specific LPR system which is geared to community on the basis of an improved Hausdorff distance. The present paper is organized as follows: Firstly, description of the overall system is discussed in section 2, Secondly, the image preprocessing and LP location are introduced in section 3. The improved hasudorff distance and symbols/characters recognition strategy are proposed in section 4. Experimental results are presented in section 5, Finally, the conclusion in section 6 . 


\section{System Structure and Workflow}

Fig.1 shows the main monitor system architecture that has been geared to community. There are four integral parts: control center, image acquisition, transmission system and display screen. In the image acquisition, we use CCD Cameras and image grabbing card to get information, the video signal will be transmitted to monitor screen directly via video switch, and the video information will be stored in image processing server. The control center decides when or what information should be written in storage medium, as well as whether to switch monitory point or to start warning system if necessary. If there occurs nonnormal case which fits the warning rules predefined, the server will start the LPR algorithm to implement the monitor, the main functions is shown in Fig2.

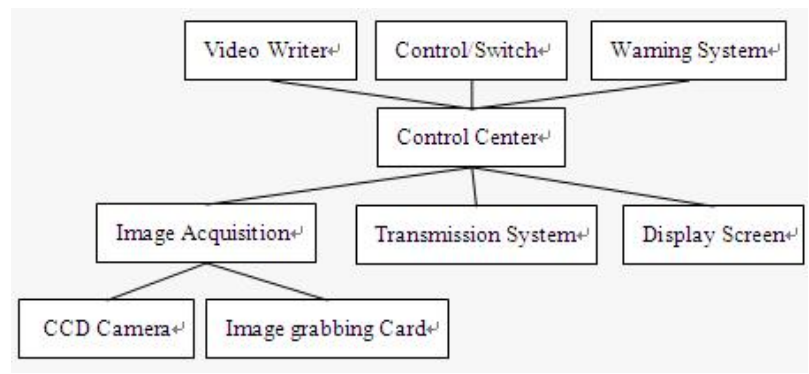

Figure 1 Monitor system structure

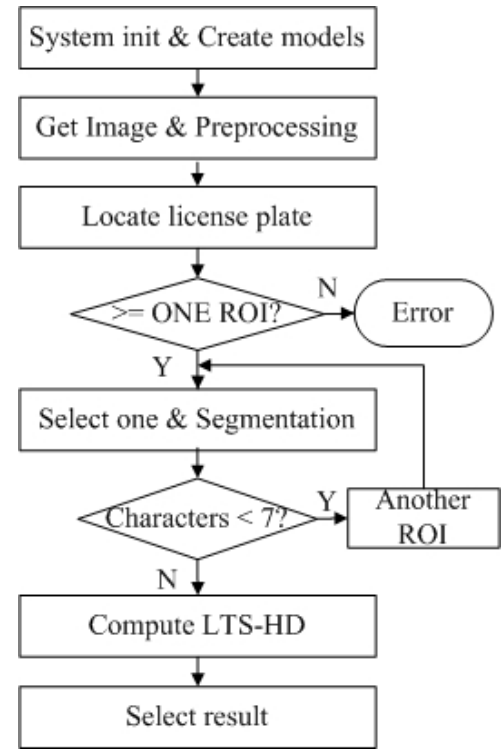

Figure 2 LPR alogrithm overview

To prepare for characters recognition, we need to set the models in advance. According to the standard for vehicle plate in china, each license plate number consists of 7 digits, of which the first symbol is a Chinese character, and the rests are letters or digits. Generally, there are about 60 Chinese characters, 26 capital letters and 10 digits. The number of symbols is limited, so the database of models can be permutated without difficulty. The size of model is $26 \times 14$, the background is white and its gray value is 255 , and the character edge is black with the gray value equaling 0 . Thus, we build the reference matching database which has 100 models approximately, and it will be loaded with the system started.

\section{Image Preprocessing and Location}

\subsection{Image preprocessing}

The result of image preprocessing has very important influence on license plate location. We use three main steps including binary image, image enhancement and denoising to complete the image preprocessing with the purpose of reducing the undesired signal and enhancing the useful information about license plate. Firstly, the colored image is transformed into gray, and then gets the binary image as the following formula (1):

$$
f(x, y)=0.3 R(x, y)+0.59 G(x, y)+0.11 B(x, y)
$$

On this basis, we use formula (2), (3), [4] to obtain the binary image. The enhancing technology is to make up for the deficiency lacking in contrast and details of image at the same time.

$$
\begin{aligned}
& p_{i}=\frac{\alpha}{\beta}\left(f_{i}-f^{\prime}\right)+f^{\prime} \\
& f^{\prime}=\frac{1}{N_{x} N_{y}} \sum_{x=0}^{N_{x}} \sum_{y=0}^{N_{y}} f(x, y)
\end{aligned}
$$

Here, $\alpha$ and $\beta$ mean enhancing factor which are relatively prime positive integers and can be regulated dynamically, $p_{i}$ represents the $i$ th gray value of converted image, $f^{\prime}$ is the mean gray value and $f(x, y)$ is a certain point gray value of original image respectively.

\subsection{License plate location}

According to traffic laws in china, the license plate has three mainly features[4], [5], [6]: 1) Color: the color of plate includes blue colored base with white colored characters, black base with white character$\mathrm{s}$, yellow base with black characters and white base with black colored characters. To improve the validity of ROI, we could utilize the color information fully to locate the plate. For instance, most civilian vehicle uses the combination of blue with white, which occupies more than $75 \%$ in community. On this occasion, 
the LP ROI should include white-blue color, and the distance between white points and blue points should be within in certain range[7], [8].

Thus, we define each point has $C_{r}, C_{g}, C_{b}$ color variable. If $C_{b}>f_{1} C_{r, g} \& C_{b}>T_{1}$, then this is a blue point; if $C_{r, g, b}<f_{2}\left(C_{r}+C_{g}+C_{b}\right) \& C_{r}+C_{g}+C_{b}>T_{2}$, then this is a white point. Here, $f_{1}, f_{2}, T_{1}, T_{2}$ are initial parameters and can be set on the basis of practical situation. 2) Structure: conventional plate has the size of $440 \mathrm{~mm}$ width by $140 \mathrm{~mm}$ height, and is always located in lower of vehicle, meanwhile the aspect ratio and character spacing are constant (each character has $45 \mathrm{~mm}$ width, $90 \mathrm{~mm}$ height, $34 \mathrm{~mm}$ character spacing between the second and the third character, $12 \mathrm{~mm}$ character spacing inter others ). 3) Texture: the chromatic transformation at horizontal direction occurs regularly with wave crests and wave troughs alternately. According to the above-mentioned three rules, the system can locate the license plate form the several ROIs quickly and efficiently. For instance, influenced by the cars decorations, there will be several LP ROIs as shown in Fig.3.

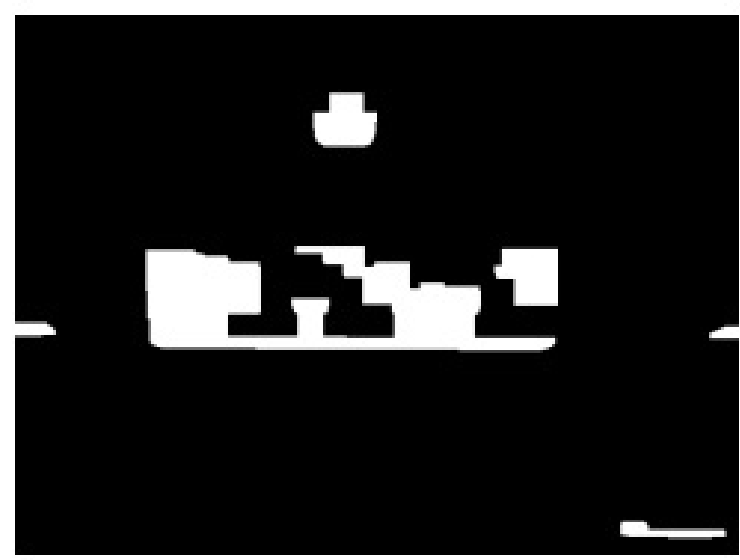

Figure 3 an example of ROIs

After determination of ROIs, the license plate location becomes easier. A slide window [9], [10], [11] is shown in Fig.4, whose width and height sizes are bigger than those of the LP size. In this window, if the number of characters is equal to or lager than 7 , it may be a good potential, otherwise, we should slide this window or discard the select ROI.

The instance of LP histogram and located plate are show in Fig.5 and Fig.6.

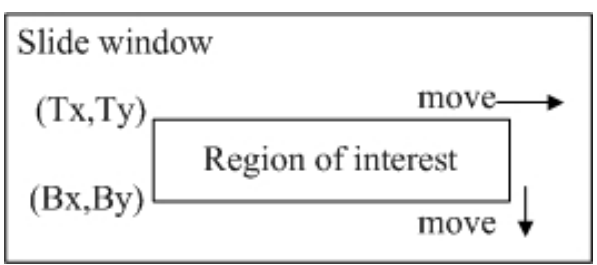

Figure 4 the slide window

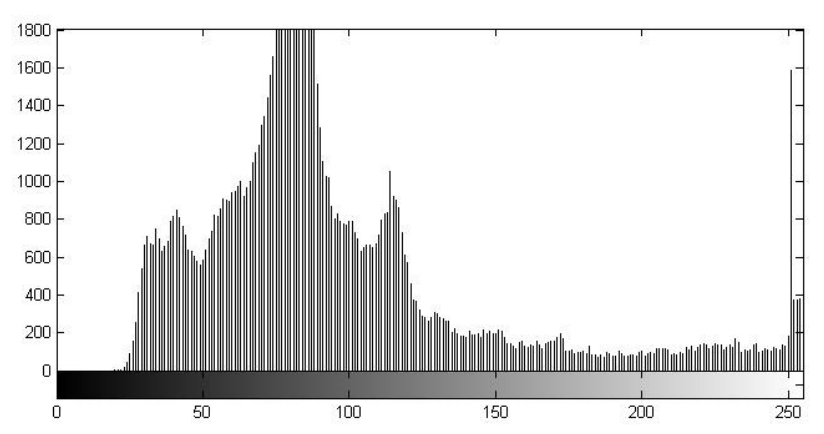

Figure 5 Example of LP histogram

\section{Segmentation and Matching}

\subsection{Character segmentation}

We should process characters segmentation [12] after getting the plate region to recognition. Wiping off some interference factors including elimination plate frame and rivets influence are very important, because the accuracy of character segmentation plays a very important role in the character recognition. Transverse projection and longitudinal projection are used in this paper. The result is shown in Fig.7.

After this preparation work, character segmentation can be done. Generally, vertical projection, template matching and connected domain segmentation are three common methods, and as vertical projection performs quickly and efficiently [5], [13], so we use this method to complete the segmentation. The brief process is listed below Fig.8.

Firstly, when we get the LP with denoising, the mean and the minimum value of vertical projection can be

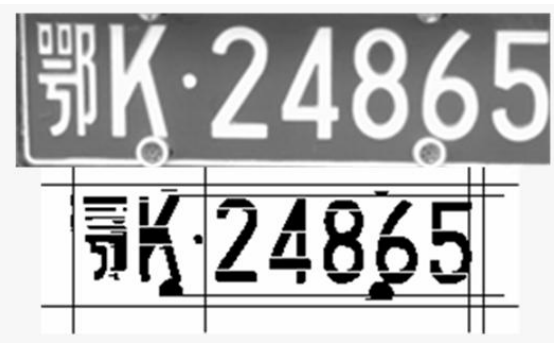

Figure 6 License plate 


\section{剻K'24865}

Figure 7 Elimination plate frame

computed, and then height and width of character is also obtained. In this process, height and width of character can be dynamically adjusted, which makes the projection more flexible. According to the character pitch, the segmentation can be done. The symbols using the vertical projection of gray value is shown in Fig.9. Furthermore, In order to improve the rate of identification and reduce the calculated complexity, we can thin each character before matching as show in Fig.10.

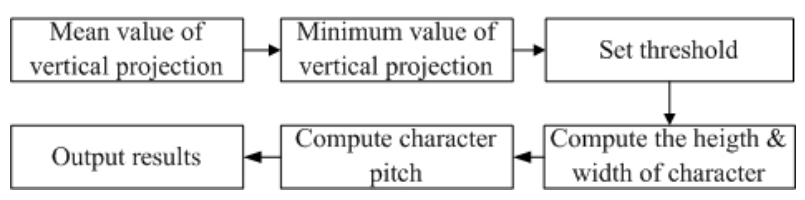

Figure 8 Segmentation process

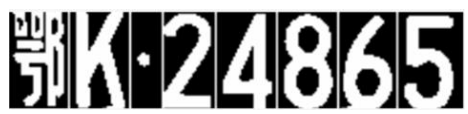

Figure 9 Segmenation

After character segmentation and thinning, we can get seven separated white colored with black colored base images, and in order to extract the feature of image, we do some normalization for the pictures such as to use uniform size for each separated characters like what Fig.11 shows.

\subsection{Matching based on improved hausdorff dis- tance}

In the past decades, several strategies have been proposed successfully in image matching. These strategies include Hidden Markov chain, neural network, Wavelet analysis and fuzzy mathematics [14], but these strategies mentioned above are mainly developed for specific application and are apt to research in laboratory. The most commonly used method for recognition is certainly based on the model matching which is one of the representative algorithms of image identification. Model matching computes distance between models and some feature vectors extracted from image that wants to detect. The minimum distance rep-

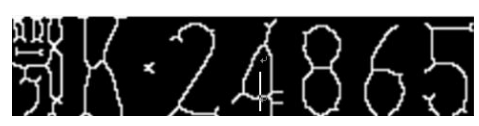

Figure 10 Thinning the characters

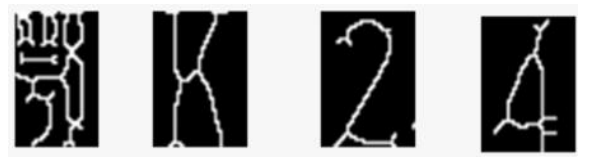

Figure 11 Separated characters

resents the compare result. Simple algorithm and reliable performance are the features of this method. The hausdorff distance computation differs from many other shape comparison methods; it is reliable even when the image contains multiple objects, noise, spurious features, and compares a portion of a model against an image [15], [16].

The Hausdorff distance [17], [18], [19] was a shape comparison metric based on binary images. It represents the difference of two point sets. For example, there are two finite point sets $A=\left\{a_{1}, \cdots, a_{m}\right\}$ and model $B=\left\{b_{1}, \cdots, b_{n}\right\}$ that the hausdorff distance of $A$ and $B$ is defined as:

$$
H(A, B)=\max [h(A, B), h(B, A)]
$$

Where

$$
h(A, B)=\max _{a \in A} \min _{b \in B}\|a-b\|
$$

And \|\| is the Euclidean norm on the points of $A$ and $B$. $h(A, B)$ is called the directed Hausdorff distance from $A$ to $B$.If $h(A, B)=d$, it means the distance between each point in set $\mathrm{A}$ and $\mathrm{B}$ is no more than $\mathrm{d}$. conversely,

$$
h(B, A)=\max _{b \in B} \min _{a \in A}\|b-a\|
$$

(6) is called the directed Hausdorff distance from B to A. HD matching is a method of feature points matching that does not re-quire to build point-to-point correspondence, but only needs to compute the similarity of two points, so it has good performance to deal with images having multi feature points or transformation. Huttenlocher et al. pro-posed the partial HD [18] measure in comparing partial portions of images containing severe occlusions or degradation. The directed distance of the partial HD [20], [21] is defined as:

$$
H_{f, r}(A, B)=\max \left[h_{f}(A, B), h_{r}(B, A)\right]
$$


$1 \leq f \leq \operatorname{sum}$ points of $A, 1 \leq r \leq$ sum points of $B$. There $h_{f}(A, B)=f_{a \in A}^{t h} \min _{b \in B}\|a-b\|$ denotes.

The $f^{\text {th }}$ ranked value of point in A to point B. Dubuisson and Jain proposed the MHD based on the average distance value in comparing the synthetic images contaminated by four types of noise [22]. The directed distance of the MHD is defined as:

$$
h_{M H D}(A, B)=\frac{1}{p} \sum_{i=1}^{p} \min \|a-b\|(i)
$$

Reference [23] present the least trimmed square (LTS) HD based on LTS which is combined with PHD and MHD, the LTS-HD is defined as:

$$
h_{L T S}(A, B)=\frac{1}{F} \sum_{i=1}^{F} \min \|a-b\|(i)
$$

It ranks value of the distance between any point in set $A$ to set $B$ by ascending order, and gets the sum of the 1th to the fth, then computes the mean value.

Here, $F$ represents $f \times N_{A}$ as in the PHD case, and $f$ as an optimal fraction whose range is from zero to one. If $\mathrm{f}$ is equal to one, then this HD computing method corresponds to the conventional MHD. This method can eliminate the influence occurred by margin points as well as doing well for zero-mean Gaussian noise.

We use the LTS-HD method to realize the matching of plate characters and models, in order to obtain the ideal matching results. The main steps of algorithm shows as:

Step1:For $i=1,2 \cdots, N ; / / \mathrm{N}$ is the number of model

Step2: $h_{L T S}\left(X, T_{i}\right)=0 ; / / T_{i}$ is the ith model

For $j=1,2 \cdots$,rows do

For $i=1,2 \cdots$, columns do

If $\mathrm{P}(\mathrm{x}, \mathrm{y})=1$ then

Search the value of point equal 1 at near-

est to $3 \times 3$ which centered

$P(x, y)$ in model

If find then

Compute $h_{L T S}\left(X, T_{i}\right)$

If not find then

Set $h_{L T S}\left(X, T_{i}\right)=h_{L T S}\left(X, T_{i}\right)+30$

Step3: Compute $h_{L T S}\left(T_{i}, X\right)$ using steps be similar to Step2

Step4: Result $=\min \left(H_{L T S(1)}, H_{L T S(2)}, \cdots, H_{L T S(N)}\right)$

\subsection{Match optimization}

In this paper, two primary methods are used to optimize the license plate match. Firstly, this algorithm is running for the community monitor, mostly LP having the same features in the same community, so, we can set different weight value for each model and color entropy. For example, aiming at a civil community of Hubei province, we can set the weight value of model 鄂equals to 1 , and others are less than 1,meanwhile, more than $75 \%$ civilian vehicles has blue and white plate, this color entropy also can set the maximum value 1 . Of course, each community has different initial set according to its actual conditions. This optimization step can improve the LP location accuracy form several ROIs and reduce the model match times.

On the other hand, there are about 100 models in reference database; in the worse case, each model will compute the hausdorff distance with every character in license plate, and then select the minimum as the match result. This whole process is very time consuming, so, it is necessary to improve the matching instantaneity. This paper uses Early Termination Method (ETM) to stop the compute process in advance. The detail of ETM is described below:

Step1:Define the translation transformation vector $T=(x, y), M, P$ represents the model and LP character respectively, $m_{M} \times n_{N}$ is the size of $M$, and $m_{P} \times n_{P}$ is the size of $P, \tau$ is the match threshold.

Here $-m_{p}+1 \leq-m_{M}-1,-n_{p}+1 \leq-n_{M}-1$.

Step2:The distance between $M$ and $P$ on the basis of $T$ is defined as: $F(x, y)=\max \left\{F_{M}(x, y), F_{P}(x, y)\right\}$

Where $F_{M}(x, y)=\max _{h, l: g M(h, l)=1} D_{m}(h-x, h-y)$, $F_{P}(x, y)=\max _{h, l: g P(h, l)=1} \widehat{D}_{P}(h+x, h+y)$.

Step3: Compute the matched points, if the number of $\widehat{D}_{P}>\tau$ is more than a certain preset value, we can terminate match in advance and select another model.

\section{Experiments and Results}

We collect 500 vehicle images that have $500 * 400$ pixels size combing SAMSUNG SCC-C7439P camera with image grabbing card CR1010E under different background and illumination intensity, then, use visual $\mathrm{C}++6.0$ and matlab as algorithm working platform, experimental results to be shown in table 1 . In our experiment results, the recognition success rate is more than $90 \%$, mostly LP can be recognized correctly and quickly, but not all character images are identified accurately, because there are some characters looking mostly like another one, such as ' ${ }^{\prime},{ }^{\prime} T^{\prime},{ }^{\prime}{ }^{\prime}$ or ${ }^{\prime} 0^{\prime},{ }^{\prime} 8^{\prime},{ }^{\prime} Q^{\prime}$ being misclassified. Therefore, some LP recognition algorithm should be run one more time or need manual interference.

\section{Conclusions}

The LPR system geared to community is realized by applying the LTS-HD algorithm, the effectiveness 
Table 1 LP recognition results

\begin{tabular}{|c|c|}
\hline LP Location number of times & Success rate \\
\hline Once/429 & $85.80 \%$ \\
\hline More than once/56 & $11.20 \%$ \\
\hline Not find /15 & $3.0 \%$ \\
\hline
\end{tabular}

is tested with many images under different illumination and noise. The results show this method has good robustness and efficiency in community monitor. Further research will focus on improving the accuracy rate and the recognition speed.

\section{Acknowledgments}

This research is partly supported by the National Natural Science Foundation of China under Grant NO. 60705012, and the Science Foundation of Hubei Prov. under Grant NO.201110821236.

\section{References}

[1] C.Anagnostopoulos, I. Anagnostopou-los, V. Loumos, and E. Kayafas, "A license plate-recognition algorithm for intelligent transportation system applications", IEEE Trans. Intell. Transp. Syst., vol. 7, no. 3, pp.377-392, 2006.

[2] Kasaei, S.H.M.,"Extraction and recognition of the vehicle license plate for passing under outside environment", Intelligence and Security Informatics Conference (EISIC2011), pp.234-237, 2011.

[3] Lihong Zheng, Xiangjian He, Bijan Samali , Laurence T. Yang, "Accuracy enhancement for License plate recognition", 10th IEEE International Conference on Computer and Information Technology $(C$ IT2010), pp.511-516, 2010.

[4] Y. Wan, X.Y. Wei, "New algorithm for preprocessing in the location of license plate image", Chinese Journal of scientific Instrument, vol.28, pp.476-479, 2007.

[5] Yifan Zhu,Han Huang,Zhenyu Xu,Yiyu He,Shiqiu Liu, "Chinese-style plate recognition based on artificial neural network and statistics", Procedia Engineering, vol.15, pp.3556-3561, 2011.

[6] Lejiang Guo, Yahui Hu, Ze Hu, Xuanlai Tang, "The edge detection operators and their application in license plate ecognition", Computational Intelligence and Software Engineering (CISE2010),pp.1-4, 2010.

[7] Wen Y, Lu Y,Yan J, Zhou Z,von Deneen, K. M. Shi P., "An algorithm for license plate recognition applied to intelligent transportation system", IEEE Transactions on Intelligent Transportation Systems, vol.12,no.3,pp.830-845, 2011.
[8] D.Renuka devi, D .KanagapushpavaUi, "Automatic license plate recognition", Computing, Сотmunications and Applications Conference (ComComAp2012), pp.117-122,2012.

[9] Danian Zheng, Yannan Zhao, Jiaxin Wang, "An efficient method of license plate location", Pattern Recognition Letters, vol.26,pp.2431-2438,2005.

[10] Yuh-Rau Wang, Wei-Hung Lin, Shi-Jinn Horng, "A sliding window technique for efficient license plate localization based on discrete wavelet transform", Expert Systems with Applications,vol.38, pp.31423146,2011.

[11] Xing Yang, Xiao-Li Hao, Gang Zhao, "License plate location based on trichromatic imaging and colordiscrete characteristic", Optik,vol.123, pp.14861491,2012.

[12] Bo Shu-kui, SUN Xin-de and DING Lin,"Method of license plate detection based on color image segmentation", Computer Science, vol.36, no.4, pp.261-267, 2009.

[13] Wenjing Jia, Huaifeng Zhang, Xiang-jian He, "Region-based license plate detection", Journal of Network and Computer Applications,vol.30, pp.1324-1333, 2007.

[14] Jianyu Zhao, Shujian Ma, Weimin Han, Yang Yang, Xudong Wang, "Research and implementation of license plate recognition technology", Control and Decision Conference (CCDC2012), pp.3768-3773, 2012.

[15] M. Lalonde, S. Foucher, L. Gagnon, "Unconstrained license plate detection using the hausdorff distance", SPIE Defense Security, Visual Information Processing XIX, Proc. SPIE, vol.7701, pp.1-7, 2010.

[16] Anagnostopoulos, C. N., Anag-nostopoulos, I., Psoroulas, I. D., Lou-mos, V., Kayafas, E, "License plate recognition from still images and video sequences: a survey", IEEE Trans. on Intelligent Transportation Systems, vol.9,no.3, pp.377-391, 2008.

[17] D. P. Huttenlocher, G. A. Klanderman, W. J. Rucklidge, "Comparing images using the hausdorff distance", IEEE Transaction on Pattern Analysis and Machine Intelligence, vol.15,no.9,pp.850-863, 1993.

[18] W. J. Rucklidge, "Efficiently locating objects using the hausdorff distance", International Journal of Computer Vision, vol.24,no.3,pp.250-271, 1997.

[19] Chen Ling-zhi, WANG Bao-baa and DONG Jianguo, "A modified algorithm for model-based matching using Hausdorff distance", Computer Technology and Development,vol.19,no.5, pp.82-85,2009.

[20] M. P. Dubuisson, A. Jai, "A modified hausdorff distance for object matching", Proceedings of International Conference on Pattern Recognition, pp.566$568,1994$. 
[21] Zhi-Qiang Zhou, Bo Wang, "A modified hausdorff distance using edge gradient for robust object matching", Image Analysis and Signal Processing, IASP 2009, pp.250-254,2009.

[22] D. G. Sim, O. K. Kwon, R H. Park, "Object matching algorithm using robust hausdorff distance measures", IEEE Transaction on Image Processing,vol.8, no.3, pp.425-429,1999.

[23] Y. Wan, X.Y. Wei, "New algorithm for preprocessing in the location of license plate image", Chinese Journal of scientific Instrument,vol.28, pp.476-479,2007. 